\title{
Segmentation Strategy of Handwritten Connected Digits (SSHCD)
}

\author{
Abdeldjalil Gattal $^{1,2}$ and Youcef Chibani ${ }^{3}$ \\ ${ }^{1}$ Université de Tébessa, Algeria \\ ${ }^{2}$ Ecole Nationale Supérieure d'Informatique (ESI), Oued Smar, Algeria \\ ab.gattal@gmail.com \\ ${ }^{3}$ Laboratoire de Communication Parlée et Traitement des Signal, \\ Faculté d' Electronique et d'Informatique, University of Sciences and \\ Technology Houari Boumedienne, Bab-Ezzouar, Algiers, Algeria \\ ychibani@usthb.dz
}

\begin{abstract}
The handwritten digit segmentation is the most important module for handwritten digit recognition, which constitutes a difficult task because of overlapping and / or connected of adjacent digits. To resolve this problem, several segmentation methods have been developed each one having its advantage and disadvantage. In this work, we propose a segmentation approach depending of the configuration link between digits. With the help of a few rules, multiple hypotheses are defined for finding the best segmentation path in order to separate two connected digits. Hence, a verification strategy is proposed in order to generate all possible segmentation-recognition hypotheses. The performance of our strategy is evaluated in terms of correct recognition rates using the confusion matrix.
\end{abstract}

Keywords: recognition, segmentation, segmentation-recognition, handwritten digits, verification strategy, Support Vector Machines.

\section{Introduction}

The handwriting recognition is interested in many applications such as automatic sorting postal mail, the automatic processing of the administrative files, or the recording the courtesy amount of the bank checks [1]. In this work, we are interested to develop a segmentation method for finding the best way of separation when the digits are connected.

Usually, the handwritten digits recognition can be done mainly in three steps. The first step facilitates further processing (smoothing or reorganization of characters, homogenization of the line thickness, etc...) in order to locate and isolate the characters more easily from each other. This step, called segmentation, [2],[3],[4],[5] consists in separating the text into elementary characters for which the limited number of characters defines the possible distinct classes. Then, a feature generation is performed on the character image for reducing the dimension of the representation and thus makes the design of the classification system. Finally, a decision function allows assigning a character image to predefined class. 
Such recognition system, the segmentation constitutes the most difficult step, which is related to several factors such as the slope of the figures, overlapping of two digits, and the joining of two consecutive digits or the default inking.

Usually, the segmentation can be conducted in considering three following situations: distinct digits and overlapped digits or connected digits. In most cases, the overlapped and connected digits are the frequent situations observed. Hence, many algorithms were proposed to separate the couples of contiguous digits. Some ones are based on contours and others on the skeleton or on the size, the number and the position of the water reservoir to deduce the potential points of cutting [6], [1], [3].

In this paper, we only are interested in the segmentation where we try to improve the performances by offer the best cutting when the digits are naturally connected. Hence, we propose a strategy based on an isolated verifier, which was responsible for detecting both over-segmentation and under-segmentation for solving the majority of the segmentation problems [8].

The paper is organized as follows. In section 2, we review the segmentation method and its strategy. The section 3 is devoted to present the experimental results. Finally, the conclusion and future work are presented in Section 4.

\section{Segmentation Method}

The segmentation consists to find the best way of cutting to isolate the digits [6],[7],[9]. However, finding the best way is not straightforward because two contiguous digits can be insulated, deformed, related by one or more contact points, partially superimposed, or on the contrary written in several pieces. Moreover, several factors such as the variability of the style and the tool of writing increase the complexity of segmentation. Fig.1 illustrates some difficult examples.

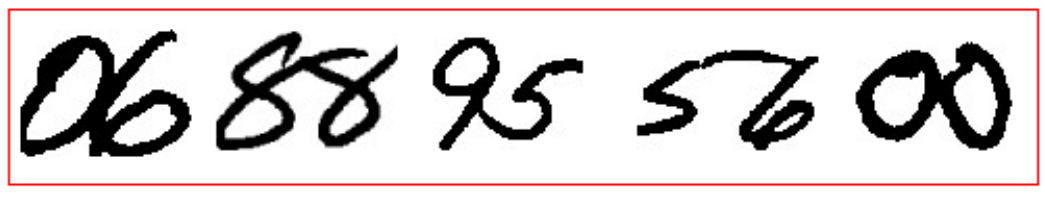

(a)

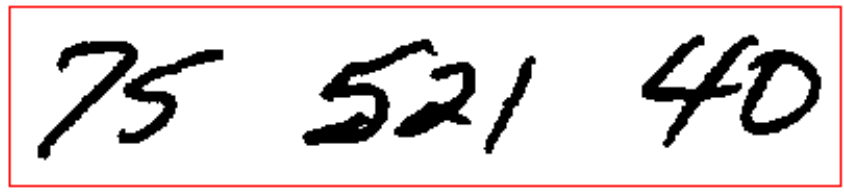

(b)

Fig. 1. Some difficult examples

(a) Connected digits (b) Overlapped digits 
In this paper, we propose an approach based on the verifications strategy in order to generate all possible segmentation-recognition hypotheses. In the following, we review how to find all possible segmentations paths, by analyzing the interconnection points [2].

\subsection{Segmentation Based on the Interconnection Points}

This method has been proposed more recently which involves analyzing the number and nature of interconnection points between two adjacent digits in order to define the optimal position for cutting a digit image couple [2]. The first step is to define the Interconnection Points (IPs) and the Bases Points (BPs) from which to start the segmentation. BPs are obtained from the extrema (minima and maxima) detected on the local contour connected components while IPs are calculated using the Freeman code according to the 8 directions in the clock-wise. Often, the connection points contain IPs, BPs or both at the same time. Hence, three hypotheses can be considered for the optimal segmentation:

- Hypothesis 1: If the Euclidean distance between the projection of the BP and the IP is lower than a threshold, the cut is made in the vicinity of the IP (Fig.2.a).

- Hypothesis 2: if the lower segment of IP is related to a upper segment of IP (or vice versa) and both IPs are near a BP, the skeleton path linking both IPs (Skeleton path) is used as part of the segmentation cut with complementary paths between BPs and IPs (Composed path) (Fig.2.b)

- Hypothesis 3: in some cases, even if there is a connection between two digits, the skeleton path does not have an IP. Thus, to avoid the under-segmentation (lack of segmentation point), the algorithm builds a path of segmentation based minimal Euclidean distance between bases points upper and bases points lower (closest points) in the middle (Fig.2.c).

\subsection{Segmentation Strategy of Connected Digits}

The main problem for separating two connected digits is the detection of the interconnection points (IP). Hence, we propose the following steps:

- $\quad$ For detecting the IP, we set up a window where the height has the same height as the original image and its width is constant. The IP is located in the middle of this width.

- For each window, if a single IP is detected, the cut is conducted in the vicinity of IP when the Euclidian distance between IP and BP (BP upper or BP lower) is lower than a threshold T1. If there are two IPs (IP upper and IP lower), then we apply the hypothesis 2 , in order to separate the two digits. To avoid undersegmentation, we apply the Hypothesis 3 in all cases.

The threshold T1 is determined through experimentation, seeking the maximum Euclidean distance between IP and BP in the case of connected digits. 


$$
\begin{gathered}
23 \cdot 23 \cdot x \cdot 23 \\
06 \cdot 06 \cdot \frac{x}{x} \cdot 06 \\
57 \cdot 57 *-.57 \\
\frac{26}{26}
\end{gathered}
$$


maximum value of the averages decision functions between the results of the segmentation hypothesis. This verification allows reducing the confusion between isolated and under-segmented characters.The diagram in Figure 4 is designed completely segmentation, recognition and verification. In the column "outputs", we can see the results of the decision function produced, for each SVM classifier [11] according to the rules (hypotheses) presented in Figure 3.

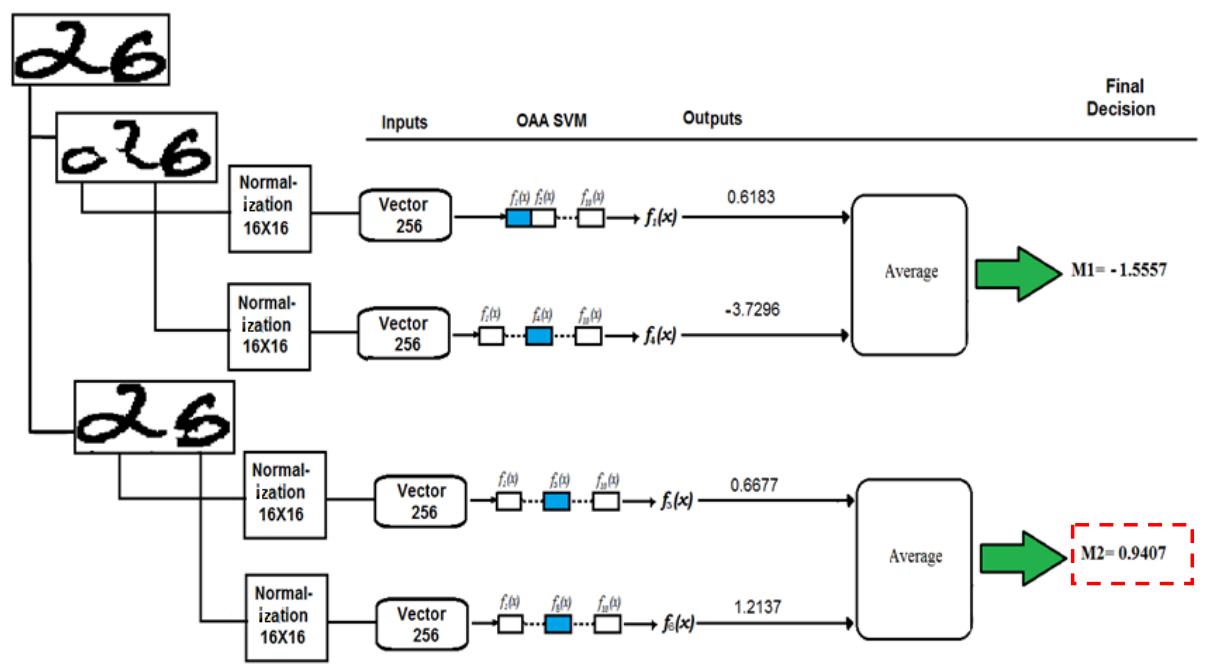

Fig. 4. Final decision for the segmentation-recognition

\section{Experimental Results}

The evaluation of a segmentation method is very subjective. It can be done in two steps. The first is to evaluate the segmentation based on a priori knowledge about the effects of segmentation. The second step is to evaluate the system for recognizing handwritten digits by integrating segmentation and classification. In our case, we use the system evaluation method for appreciate the quality of the segmentation on a database of NIST SD19 [10].

The NIST SD19 database is divided into two parts: the first consisting of 5000 digits is used for learning and the second 500 digits is used for testing. The test database contains 250 digits linked. Each digit is normalized to $16 \times 16$ pixels after segmentation.

The recognition module is based on a SVM classifier with RBF kernel. The multiclass SVM implementation is based on the approach "one against all". It involves using a binary classifier by category. The SVM and RBF parameters are fixed to $\mathrm{C}=10$ and $\sigma=10$, respectively. Furthermore, the threshold $\mathrm{T} 1$ is fixed experimentally to 7 .

The evaluation is conducted without feature extraction in order to evaluate the quality of segmentation. This evaluation is illustrated in the confusion matrix reported in Table 1. 
Table 1. Recognition rates obtained for each class

\begin{tabular}{|c|c|c|c|c|c|c|c|c|c|c|c|c|}
\hline & \multicolumn{10}{|c|}{ Reference Classes } & \\
\hline & & 0 & 1 & 2 & 3 & 4 & 5 & 6 & 7 & 8 & 9 & \\
\hline \multirow{10}{*}{ 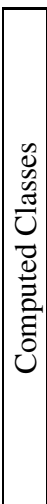 } & 0 & 81.08 & \begin{tabular}{|l|}
2.70 \\
\end{tabular} & 2.70 & 2.70 & 2.70 & & 2.70 & & 5.41 & & \\
\hline & 1 & & 100.00 & & & & & & & & & \\
\hline & 2 & & & 77.27 & & & & & 9.09 & 4.55 & & \\
\hline & 3 & & & & 66.67 & & & & & 4.76 & & \\
\hline & 4 & & & & & 81.25 & 6.25 & & & & & \\
\hline & 5 & & & & & & 91.18 & 8.82 & & & & \\
\hline & 6 & & & 6.25 & & & & 81.25 & & & & \\
\hline & 7 & & & & & & & & 100 & & & \\
\hline & 8 & & & & & & & & & 88.89 & & \\
\hline & 9 & & & & & 5.00 & & & & & 95.00 & \\
\hline & \multicolumn{11}{|c|}{ Overall Rate of SSCHD method. } & 86.26 \\
\hline
\end{tabular}

We can note that some digits are not recognized, which are considered as rejected by the SVM classifier. Furthermore, we can note that some digits are difficult to recognize specifically the couples $\left(' 0,{ }^{\prime}, 8^{\prime}\right),\left(2^{\prime}, 7^{\prime}\right)$ and $\left({ }^{\prime}{ }^{\prime}, 8^{\prime}\right)$. These digits are more problematic for recognition since they are sometimes visually very similar. The decision is difficult even for a human operator.

\section{Conclusion and Future Work}

The objective of this paper is the presentation of a strategy for segmenting connected handwritten digits, in order to find the best cut to isolate two adjacent digit images.

The method allows improving the performances and resolving many problems of connected digits. It uses conjointly segmentation-verification for finding the way of cutting of the connected digits.

The first results obtained are encouraging since we can manage to follow, with a weak error of detection, all the possible ways of segmentation. This combination uses few rules and has the advantage of providing a correct segmentation in the most cases.

For future work, we try to complete the system by adding an analysis module for generating the features. For a complete validation of the system, we also try to evaluate the system on a larger database.

\section{References}

1. Dimauro, G., Impedovo, S., Pirlo, G., Salzo, A.: Automatic Bankcheck processing: A New Engineered System. International Journal of Pattern Recognition and Artificial Intelligence 11(4), 467-504 (1997)

2. Vellasques, E., Oliveira, L.S., Britto Jr., A.S., Koerich, A.L., Sabourin, R.: Filtering segmentation cuts for digit string recognition. Pattern Recognition 41(10), 3044-3053 (2008) 
3. Congedo, G., Dimauro, G., Impedovo, S., Pirlo, G.: Segmentation of Numeric Strings. In: Proc. of Third Int. Conf. on Document Analysis and Recognition, Canada, pp. 1038-1041. IEEE Computer Society, Montreal (1995)

4. Jang, B.K., Chin, R.T.: One-pass parallel thinning: Analysis, properties, and quantitative evaluation. IEEE Transactions on Pattern Analysis and Machine Intelligence 14(11), 1129-1140 (1992)

5. Shridhar, M., Badreldin, A.: Recognition of Isolated and Simply Connected Handwritten Numerals. Journal of Pattern Recognition 19(1), 1-12 (1986)

6. Ayat, N.E., Cheriet, M., Suen, C.Y.: Un système neuro-flou pour la reconnaissance de montants numériques de chèques arabes. Colloque international francophone sur l'écrit et le document, Montréal, Québec, Canada, pp. 03-07 (2000)

7. Hussein, K.M., Agarwal, A., Gupta, A., Wang, P.S.P.: A knowledge-based algorithm for enhanced recognition of handwritten courtesy amounts. Pattern Recognition 32, 305-316 (1999)

8. Oliveira, L.S., Sabourin, R., Bortolozzi, F., Suen, C.Y.: A modular system to recognize numerical amounts on Brazilian bank cheques. In: Proc. of 6th International Conference on Document Analysis and Recognition (ICDAR), Seattle, USA, pp. 389-394. IEEE Computer Society, Los Alamitos (2001)

9. Fujisawa, H., Nakano, Y., Kurino, K.: Segmentation Methods for Character Recognition: From Segmentation to Document Structure Analysis. Proceedings of the IEEE 80(7), 2128, 1079-1091 (1996)

10. Grother. P.J.: NIST Special Database 19; Handprinted Forms and Characters Database. National Institute of Standards and Technology, NIST (1995)

11. Schölkopf, B., Burges, C., Vapnik, V.: Extracting support data for a given task. In: KDD 1995, pp. 252-257 (1995) 